\title{
Imaging, screening and remote sensing of photosynthetic activity and stress responses
}

\author{
Kaori Kohzuma ${ }^{1} \cdot$ Kintake Sonoike $^{2} \cdot$ Kouki Hikosaka ${ }^{1}$
}

Published online: 21 June 2021

(c) The Botanical Society of Japan 2021

Keywords Remote sensing $\cdot$ Photosynthesis $\cdot$ Imaging

All lives rely on photosynthesis. It provides energy and carbohydrates not only to photosynthetic organisms themselves but also to heterotrophic organisms through the food web. It is also one of the most important processes in global carbon cycling. Improvement of photosynthesis is a key to solving problems that human society faces. Crop management and breeding of cultivars with higher photosynthetic activities may contribute to increasing crop yields, which are needed to meet rapid population growth. Enhancement of carbon sequestration in terrestrial ecosystems may be one of the effective ways for a low-carbon economy.

The spatial scale of photosynthetic measurements varies greatly among target organisms. The gas exchange method $\left(\mathrm{CO}_{2}\right.$ or $\left.\mathrm{O}_{2}\right)$ is the most reliable and frequently used. From cellular (or chloroplast) to leaf and individual scales, samples are put into or clamped with an assimilation chamber, and gas exchange is directly determined with an infrared gas analyzer or $\mathrm{O}_{2}$ electrode. In particular, commercial portable gas exchange measurement systems can be used for leaf photosynthesis determination even in the field. For vegetation scales, the eddy covariance method directly determines $\mathrm{CO}_{2}$ exchange between the vegetation and atmosphere using an infrared gas analyzer and anemometer installed on a flux tower. However, these methods have some shortcomings.

Kaori Kohzuma

kohzuma@googlemail.com

Kouki Hikosaka

hikosaka@tohoku.ac.jp

1 Graduate School of Life Sciences, Tohoku University, Sendai, Miyagi 980-8578, Japan

2 Faculty of Education and Integrated Arts and Sciences, Waseda University, Shinjuku-ku, Tokyo 162-8480, Japan
First, most of these methods have some influence on the target. For example, the leaf gas exchange system needs to clamp the target leaf, which may physically damage the leaf. Second, the measurement is often time-consuming as it waits for the gas exchange in the system to become stable. Third, such methods hardly identify heterogeneity of photosynthetic activity in the system. Such shortcomings are critical, especially when we want to identify individuals that have unusual photosynthetic activity in agricultural field management, mutant screening, or breeding. Furthermore, as the target scale increases, determining its gas exchange becomes technically more difficult.

Optical observations are an alternative method to assess photosynthesis without the problems mentioned above. As the photosynthetic apparatus involves various pigment molecules, each of which has unique absorption spectra, reflectance spectra from plants include various information related to photosynthesis. Furthermore, the yield of fluorescence emitted by photosystem II (PSII) chlorophylls (Chl fluorescence) is very informative because it changes depending on the status of PSII; it decreases when the energy allocation to PSII photochemical reaction (photochemical quenching) or heat dissipation increases (non-photochemical quenching). Various methods have been developed to assess photosynthetic status from optical information, such as the pulseamplitude modulation (PAM) system and the Fraunhofer line depth method. Nowadays, we can obtain photosynthetic information by optical observations at various spatial scales using cameras installed on a microscope observation tower, unmanned aerial vehicle, airplane, satellite, etc.

Time scale is also an important aspect of obtaining different information. Short-term analyses are useful to know the status of the photosynthetic apparatus. There are multiple photosynthetic processes that are inactive in the dark. 
Such processes are activated after illumination, altering energy partitioning to PSII, which can be detected as the changes in Chl fluorescence yield. As the time constant for the activation differs among the processes from millisecond to minutes, $\mathrm{Chl}$ fluorescence transitions are useful to assess the status of such processes. We may obtain information on the process that is suppressed by stress or mutation, which would contribute to stress physiology and breeding science. In ecology, long-term assessment is indispensable in understanding the dynamics of vegetation and its response to climate change because many ecological processes, such as succession and evolution, take a long time to change. Spectroradiometers installed on towers and satellites are useful for this purpose.

In this special issue of JPR Symposium "Imaging, screening, and remote sensing of photosynthetic activity and stress responses", we would like to provide several methods for measuring photosynthesis at various scales with four review articles and three original papers, which are arranged in order of the spatial-temporal scale. As you begin to read this issue, your perspective is in a bacterium, but eventually it shifts to a global perspective viewed from space. We urge you to read in order from the beginning rather than picking individual papers.

Imaging chlorophyll fluorescence can obtain rapid and reliable data simultaneously, expecting that it is an effective tool for screening mutants of photosynthesis-related genes. Ogawa and Sonoike (2021) review chlorophyll fluorescence assay, including a method for tracking electron transfer in Photosystem II from the rapid response of chlorophyll fluorescence on the order of milliseconds after light exposure. The actual screening of higher plants and algal cells conducted in the past and the mutants acquired through such the technique (e.g., Ozaki et al., 2007) that have contributed significantly to the study of photosynthesis are introduced.

In order to solve various social problems by applied photosynthesis research, it is necessary to understand the molecular mechanisms of photosynthesis and its measurement devices. Cruz and Avenson (2021) describe the methods and the principles of non-destructive measurements of plant photosynthesis, such as chlorophyll fluorescence analysis, in vivo spectroscopy, and gas exchange measurements with the fundamental photosynthetic mechanisms. These measurement methods have a long history and are used in many studies. However, the interpretation of these data, new measurements, or parameters are constantly evolving and provided (Cruz et al. 2016).

Imaging photosynthesis is not limited to screening mutants to understand their mechanisms but can be extended to the agricultural field. Kohzuma et al. (2021) used a hyperspectral camera to detect and visualize environmental stress in crops from the photochemical reflectance index (PRI). PRI is a parameter that detects xanthophylls, photosynthetic pigments that change under environmental stress, from the reflected signal at $531 \mathrm{~nm}$ (Kohzuma and Hikosaka 2018; Peñuelas et al. 1995). While previous PRI measurements obtained baselines in the dark, they proposed a method to correct PRI in the light and extended it to an on-farm stress detection demonstration.

Spectral observation is a primary measurement method for remote sensing in the field. Solar-induced chlorophyll fluorescence (SIF) and PRI are known as spectral signals that are related to the photosynthetic properties of plants. Hikosaka and Tsujimoto (2021) theoretically discuss how SIF and PRI are related to photosynthetic rates. $\mathrm{CO}_{2}$ assimilation rates can be estimated from either SIF and PRI with some assumptions, but such assumptions are not necessarily held when leaves are severely photoinhibited (Hikosaka 2021). $\mathrm{CO}_{2}$ assimilation rates of such leaves can be assessed with a model in which both SIF and PRI are simultaneously used (Hikosaka and Noda 2019). This model may be useful for a more accurate assessment of the photosynthetic activity of vegetations in changing environments.

SIF can be evaluated using the Fraunhofer line spectra (Fraunhofer line depth method; FLD). However, it has been suggested that the quantification of SIF requires a high-resolution spectroradiometer with full-width at half maximum $(\mathrm{FWHM})<0.3 \mathrm{~nm}$, and low-resolution spectroradiometers are not useful (Meroni and Colombo 2006). Nakashima et al. (2021) propose a new method, termed aFLD, to calculate a reasonable SIF from a spectroradiometer with low spectral resolution. This enables us to extract SIF values from past observation data accumulated in various vegetation sites on a decade scale. In the paper, SIF is obtained from spectral reflectance data observed in deciduous broadleaf forests for about 10 years (Muraoka et al. 2013) using the aFLD method. The estimated SIF was highly correlated with SIF estimated from satellite observations (GOSIF).

Unmanned aerial vehicle (UAV) -based remote sensing is beneficial for large-scale agriculture where wide-area monitoring is required. However, it is difficult to acquire accurate data with spatial continuity using UAV observations alone. Yu et al. (2021) attempt to obtain more accurate reflectance data in an apple orchard by mixing multispectral data acquired by UAV with hyperspectral data at the ground surface. Furthermore, they have obtained a more correlated inverted reflectance from the measured reflectance by comparing two models.

Remote sensing by earth observation satellites is an excellent tool for observing global ecosystems on a large temporal and spatial scale. If the amount of photosynthetic production in terrestrial ecosystems can be accurately measured from satellites and the $\mathrm{CO}_{2}$ balance can be predicted, it may lead to a more accurate prediction of climate change. However, it is difficult to predict them without linking ecological processes in forest ecosystems and optical data from satellites. 
In order to understand the mechanisms that link the two, it is helpful to scale up from single-leaf to the larger. Noda et al. (2021a) review the plant ecological processes that influence the optical properties of the measurable forest canopy in anticipation of optical remote sensing of forest ecosystems using Earth observation satellites (Noda et al. 2021b).

We have a variety of options for photosynthesis measurements, but we also understand the problems. Yu et al. (2021) have used a combination of UAV observations and another method, implying that it is difficult to obtain accurate values by only remote sensing. Understanding various measurement methods, theories, and the advantages and disadvantages of each are expected to link to the development of new technology and new knowledge. The integration and constant updating of plant physiological information provided by plant scientists and eco-physiological information provided by ecologists would resolve food and global environmental problems (Osmond and Chow 1988). We expect this special issue to be a good opportunity to widely disseminate photosynthesis measurement methods with many researchers and connect researchers with their respective research fields.

\section{References}

Cruz JA, Avenson T (2021) Photosynthesis: a multiscopic view. J Plant Res. https://doi.org/10.1007/s10265-021-01321-4

Cruz JA, Savage LJ, Zegarac R, Hall CC, Satoh-Cruz M, Davis GA, Kovac WK, Chen J, Kramer DM (2016) Dynamic environmental photosynthetic imaging reveals emergent phenotypes. Cell Syst 22:365-377

Hikosaka K (2021) Photosynthesis, chlorophyll fluorescence and photochemical reflectance index in photoinhibited leaves. Funct Plant Biol. https://doi.org/10.1071/FP20365

Hikosaka K, Noda HM (2019) Modeling leaf $\mathrm{CO}_{2}$ assimilation and Photosystem II photochemistry from chlorophyll fluorescence and the photochemical reflectance index. Plant Cell Environ 42:730-739

Hikosaka K, Tsujimoto K (2021) Linking remote sensing parameters to $\mathrm{CO}_{2}$ assimilation rates at a leaf scale. J Plant Res. https://doi. org/10.1007/s 10265-021-01313-4

Kohzuma K, Hikosaka K (2018) Physiological validation of photochemical reflectance index (PRI) as a photosynthetic parameter using Arabidopsis thaliana mutants. Biochem Biophys Res Commun 498:52-57

Kohzuma K, Tamaki M, Hikosaka K (2021) Corrected photochemical reflectance index (PRI) is an effective tool for detecting environmental stresses in agricultural crops under light conditions. J Plant Res. https://doi.org/10.1007/s10265-021-01316-1

Meroni M, Colombo R (2006) Leaf level detection of solar induced chlorophyll fluorescence by means of a subnanometer resolution spectroradiometer. Remote Sens Environ 103:438-448

Muraoka H, Noda HM, Nagai S, Motohka T, Saitoh TM, Nasahara KN, Saigusa N (2013) Spectral vegetation indices as the indicator of canopy photosynthetic productivity in a deciduous broadleaf forest. J Plant Ecol 6:393-407

Nakashima N, Kato T, Morozumi T, Tsujimoto K, Akitsu TK, Nasahara KN, Maruyama S, Muraoka H, Noda HM (2021) Area-ratio Fraunhofer Line Depth (aFLD) method approach to estimate solar-induced chlorophyll fluorescence in low spectral resolution spectra in a cool-temperate deciduous broadleaf forest. J Plant Res. https://doi.org/10.1007/s10265-021-01322-3

Noda HM, Muraoka H, Nasahara KN (2021a) Plant ecophysiological processes in spectral profiles: perspective from a deciduous broadleaf forest. J Plant Res. https://doi.org/10.1007/ s10265-021-01302-7

Noda HM, Muraoka H, Nasahara KN (2021b) Phenology of leaf optical properties and their relationship to mesophyll development in cool-temperate deciduous broad-leaf trees. Agric for Meteorol 297:108236

Ogawa T, Sonoike K (2021) Screening of mutants using chlorophyll fluorescence. J Plant Res. https://doi.org/10.1007/ s10265-021-01276-6

Osmond CB, Chow WS (1988) Ecology of photosynthesis in the sun and shade: summary and prognostications. Aust J Plant Physiol $15: 1-9$

Ozaki H, Ikeuchi M, Ogawa T, Fukuzawa H, Sonoike K (2007) Large scale analysis of chlorophyll fluorescence kinetics in Synechocystis sp. PCC 6803: Identification of the factors involved in the modulation of photosystem stoichiometry. Plant Cell Physiol 48:451-458

Peñuelas J, Filella I, Gamon JA (1995) Assessment of photosynthetic radiation-use efficiency with spectral reflectance. New Phytol 131:291-296

Yu R, Zhu X, Bai X, Tian Z, Jiang Y, Yang G (2021) Inversion reflectance by apple tree canopy ground and unmanned aerial vehicle integrated remote sensing data. J Plant Res. https://doi.org/10. 1007/s10265-020-01249-1

Publisher's Note Springer Nature remains neutral with regard to jurisdictional claims in published maps and institutional affiliations. 